\title{
The prognostic value of residual coronary stenoses after functionally complete revascularisation
}

\author{
Dennis T. L. Wong ${ }^{1,2}$ \\ ${ }^{1}$ Monash Cardiovascular Research Centre, Department of Medicine (Monash Medical Centre), Monash University and Monash Heart, Monash \\ Health, Clayton, 3168 VIC, Australia; ${ }^{2}$ South Australian Medical Research Institute (SAHMRI), Adelaide, Australia \\ Correspondence to: Dennis T. L. Wong, MBBS (Hons), MD, PhD. Monash Cardiovascular Research Centre, Department of Medicine \\ (Monash Medical Centre), Monash University and Monash Heart, Monash Health, 246 Clayton Road, Clayton, 3168 VIC, Australia. \\ Email: drdenniswong@yahoo.com.au. \\ Provenance: This is a Guest Editorial commissioned by Section Editor Yue Liu, MD (Department of Cardiology, The First Affiliated Hospital of \\ Harbin Medical University, Harbin, China). \\ Comment on: Kobayashi Y, Nam CW, Tonino PA, et al. The Prognostic Value of Residual Coronary Stenoses After Functionally Complete \\ Revascularization. J Am Coll Cardiol 2016;67:1701-11.
}

\begin{abstract}
Fractional flow reserve (FFR) has become the gold standard for functional assessment of coronary artery stenosis. Studies have confirmed the superiority of FFR guided percutaneous coronary intervention (PCI) compared to angiography guided PCI. Due to the high cost of FFR, it is not economically viable for FFR to be incorporated into every routine invasive coronary angiography. As a result, visual estimation of diameter stenosis on invasive coronary angiography still remains the cornerstone for decision making regarding revascularisation treatment for patients. This is despite recent studies questioning the "visual functional mismatch" between diameter stenosis and FFR in $57 \%$ of patients with non-left main stenosis. In patients with multivessel disease, complete revascularisation leads to improved long term outcomes. However, some lesions classified as significant by angiography may not be functionally significant. Kobayashi and colleagues demonstrated that after functionally complete revascularization with FFR guidance, residual angiographic lesions that are not functionally significant do not reflect residual ischemia or predict a worse outcome, supporting functionally complete, rather than angiographically complete, revascularization.
\end{abstract}

Keywords: Fractional flow reserve (FFR); prognosis; coronary stenosis; angiography; SYNTAX score

Submitted Aug 09, 2016. Accepted for publication Nov 29, 2016.

doi: $10.21037 / \mathrm{cdt} .2017 .01 .06$

View this article at: http://dx.doi.org/10.21037/cdt.2017.01.06

Fractional flow reserve (FFR) has become the gold standard for functional assessment of coronary artery stenosis. Studies have confirmed the superiority of FFR guided percutaneous coronary intervention (PCI) compared to angiography guided PCI. Due to the high cost of FFR, it is not economically viable for FFR to be incorporated into every routine invasive coronary angiography. As a result, visual estimation of diameter stenosis on invasive coronary angiography still remains the cornerstone for decision making regarding revascularisation treatment for patients. This is despite recent studies questioning the "visual functional mismatch" between diameter stenosis and FFR in 57\% of patients with non-left main stenosis. In patients with multivessel disease, complete revascularisation leads to improved long term outcomes. However, some lesions classified as significant by angiography may not be functionally significant. Kobayashi and colleagues demonstrated that after functionally complete revascularization with FFR guidance, residual angiographic lesions that are not functionally significant do not reflect residual ischemia or predict a worse outcome, supporting functionally complete, rather than angiographically complete, revascularization. 


\section{Perspective}

Myocardial ischaemia is an important risk factor for adverse outcome (1). The role of PCI is to reduce the burden of ischaemia. The FAME study had demonstrated that FFR guided PCI is superior to angiography guided PCI and this translates to improvement in clinical outcome (2). Although guidelines have recommended demonstration of ischaemia non-invasively before revascularisation treatment, this is still underutilised in clinical practice. Instead, visual estimation of diameter stenosis on invasive coronary angiography still remains the cornerstone for decision making regarding revascularisation treatment for patients. However, multiple studies have demonstrated discrepancy in functional significance of coronary stenosis between diameter stenosis and the 'gold-standard' FFR (3). Nevertheless, this should not necessarily spell the end of angiography guided PCI because utilisation of FFR for all routine coronary angiography would not be economically viable. In addition to the substantial cost of FFR wires, the added time of at least 5-10 minutes per vessel for FFR interrogation limits its use for all routine coronary angiography. We however do have to acknowledge the limitations of diameter stenosis alone to predict functional significance of coronary artery stenosis. The pressure drop across epicardial coronary arteries is influenced not only by atherosclerotic stenoses but also by volumetric coronary blood flow (4). As defined by Poiseuille's law of fluid dynamics, pressure gradient is influenced by coronary blood flow and viscosity, minimum radius, and lesion length. In effect, the gradient across coronary stenoses is inversely proportional to the fourth power of the lesion radius $\left(r^{4}\right)$ and is proportional to lesion length (5). As functional significance of coronary artery stenosis is determined by multiple factors, it is not surprising that diameter stenosis alone is a poor predictor. One recent study had combined a few key predictors of pressure gradient across coronary stenosis into the DILEMMA score and showed that it is superior in predicting functional significance of coronary artery stenosis than the individual indices alone (6).

This article "The Prognostic Value of Residual Coronary Stenoses After Functionally Complete Revascularization" by Kobayashi and colleagues again highlights the limitations of angiographic assessment of diameter stenosis (7). The authors performed a substudy of the FAME trial evaluating the residual SYNTAX score (RSS) and SYNTAX revascularisation index (SRI) which are angiographic markers of completeness of revascularisation for patients with multivessel coronary artery disease. Only patients who have had functional guided PCI were included in this study and there wasn't a control group. At 1 year, major adverse cardiac events (MACE) occurred in $53(12.4 \%)$ patients. RSS and SRI did not predict MACE and the result was the same in the 2-year outcome analysis. The study concluded that after functionally complete revascularisation with FFR guidance, residual angiographic lesions that are not functionally significant do not reflect ischaemia or predict a worse outcome. The findings support a functionally complete rather than angiographically complete revascularisation. The SYNTAX score is an angiographic marker of lesion complexity, not necessarily the functional significance. Coronary lesions with $\geq 50 \%$ diameter stenosis on visual estimation are defined as significant on SYNTAX score. A 50\% diameter stenosis is not surprisingly a poor predictor of functional significance as highlighted by Park and colleagues recently who reported the "mismatches" in $57 \%$ of non-left main lesions between angiographic diameter stenosis $>50 \%$ and FFR $>0.8$ (3). This study provides us with an important lesson. It highlights that cardiologists should move away from purely looking at the diameter stenosis to guide revascularisation strategy. Consideration of the length of the lesion as well as the area of myocardium subtended by the stenosis is also important. In patients with multi-vessel disease, there may be lesions which are angiographically significant but not necessarily functionally significant. There should be a low threshold of using FFR to assess the functional significance of every lesion in this instance. Pursue of functional complete revascularisation rather than angiographic complete revascularisation may be a better strategy but confirmation of the study findings in a larger study with a control arm would be ideal.

\section{Acknowledgements}

None.

\section{Footnote}

Conflicts of Interest: The author has no conflicts of interest to declare.

\section{References}

1. Shaw LJ, Iskandrian AE. Prognostic value of gated 
myocardial perfusion SPECT. J Nucl Cardiol 2004;11:171-85.

2. Tonino PA, De Bruyne B, Pijls NH, et al. Fractional flow reserve versus angiography for guiding percutaneous coronary intervention. N Engl J Med 2009;360:213-24.

3. Park SJ, Kang SJ, Ahn JM, et al. Visual-functional mismatch between coronary angiography and fractional flow reserve. JACC Cardiovasc Interv 2012;5:1029-36.

4. Bache RJ, Schwartz JS. Effect of perfusion pressure distal to a coronary stenosis on transmural myocardial blood flow. Circulation 1982;65:928-35.

5. Kimball BP, Dafopoulos N, LiPreti V. Comparative

Cite this article as: Wong DT. The prognostic value of residual coronary stenoses after functionally complete revascularisation. Cardiovasc Diagn Ther 2017;7(Suppl 2):S63S65. doi: 10.21037/cdt.2017.01.06 evaluation of coronary stenoses using fluid dynamic equations and standard quantitative coronary arteriography. Am J Cardiol 1989;64:6-10.

6. Wong DT, Narayan O, Ko BS, et al. A novel coronary angiography index (DILEMMA score) for prediction of functionally significant coronary artery stenoses assessed by fractional flow reserve: A novel coronary angiography index. Am Heart J 2015;169:564-71.e4.

7. Kobayashi Y, Nam CW, Tonino PA, et al. The Prognostic Value of Residual Coronary Stenoses After Functionally Complete Revascularization. J Am Coll Cardiol 2016;67:1701-11. 